\title{
PHOTOELECTRIC ROCKET SPECTRA AT 10 Å RESOLUTION
}

\author{
THEODORE P.STECHER \\ NASA, Goddard Space Flight Center, Greenbelt, Md., U.S.A.
}

\begin{abstract}
Spectral scans of a number of early-type stars have been made with a three-channel spectrophotometer attached to a $32 \mathrm{~cm}$ telescope. The telescope was pointed at the individual program stars with an accuracy of at least $20 \mathrm{sec}$ of arc. The spectral resolution was $10 \AA$ over the range $1150 \AA$ to $4000 \AA$. The data were recorded in digital form and processed in a computer.

The profile of hydrogen Ly-a, as smeared by the exit slit, was obtained for $\alpha \mathrm{Lyr}, \alpha \mathrm{CMA}, \beta$ Ori, and $\beta$ Tau. For the earlier-type stars observed the Ly- $\alpha$ line of the star is masked by interstellar hydrogen absorption. For $\zeta$ Oph and $\zeta$ Per the Ly- $\alpha$ absorption is considerably larger than for the other early-type stars. The identification of lines is relatively simple for the light elements but becomes more difficult with the heavier ions since there are many more weaker lines and $10 \AA$ is then insufficient resolution. Extreme P Cygni profiles are present in the earliest stars and are interpreted as mass loss at high velocity.
\end{abstract}

A full description appears in Astrophys. J. 159, Feb. 1970.

\section{Discussion}

Jenkins: At this time would you care to quote measurements of the Lyman-absorption equivalent widths in your spectra?

Stecher: I have not measured them in detail because of the difficulty in assigning the position of the continuum. Qualitatively only $\zeta$ Oph and $\zeta$ Per have large equivalent widths that are interstellar. The rest are in agreement with your measures. One indicates a low hydrogen abundance.

Jenkins: I had the impression that the continuum was reasonably well defined on either side of Lyman- $x$ in some of your tracings. Since a few of these stars have not been observed at Lyman- $\alpha$ by other investigations, it might be useful to add your information to the collection of Hi column densities in various directions.

Stecher: Yes, I plan to in the near future.

Morton: I believe you also have a scan of, Lyr. Does it show the extensive line blanketing in the 2000 to $3000 \AA$ region found in $、 \mathrm{CMa}$ ?

Stecher: a Lyr appears to have less blanketing than a CMa. A comparison will be made soon.

Carruthers: Would you care to comment on the relative velocity shifts in the P Cygni line profiles in $\gamma$ Vel as compared to $\zeta$ Pup?

Stecher: They appear to be about half that of $\zeta$ Pup. I believe you and Morton can measure this better than I can.

Underhill: The fact that $\gamma^{2}$ Vel is classified as WC8 and $\zeta$ Pup as O5 $\mathrm{f}$ indicates that the shell of $\gamma^{2}$ Vel is considerably more dense than that of $\zeta$ Pup.

Stecher: Yes, I agree. This makes it most useful to compare the two and then attempt to explain the difference.

Houziaux: We have seen many lines in your spectra, despite the quoted resolving power of $10 \AA$. Could you state what is the shape of the instrumental profile?

Stecher: The geometric slit width. 